\title{
Rotational dynamics of magnetic nanoparticles in different matrix systems
}

\begin{abstract}
Dynamic magnetic measurements on magnetic nanoparticle (MNP) samples have been widely used for the determination of structural MNP parameters as well as for the realization of bioassays. On the other hand, proposed that the MNPs are thermally blocked, i.e., that the dynamics are dominated by the Brownian rotation, and knowing the distribution of their hydrodynamic size, information on the matrix properties can be obtained. In contrast to conventional rheology, the local environment of the MNPs is sensed on the nanoscale so that important information on the embedding of MNPs in the matrix and thus the particle-matrix interaction is obtained. Depending on the characteristic length scales of the matrix and the size of the MNPs, rheological parameters, such as viscosity and shear modulus, derived from nanorheological measurements can differ from the values obtained from conventional rheology. To measure the MNP dynamics, different experimental techniques can be applied. In this contribution, the focus lies on ac susceptometry and fluxgate magnetorelaxometry. The analysis of the complex ACS spectra is generally carried out within a modified Debye model. Different approaches for the estimation of rheological parameters from the complex ACS spectra will be presented. Two model systems will exemplarily be discussed in detail. As a Newtonian matrix system, water-glycerol mixtures were studied. It is demonstrated that the dynamic viscosity can accurately be estimated from ACS measurements on well thermally blocked single-core as well as on multicore MNP systems, which include Brownian and Néel dynamics. As a viscoelastic matrix system, aqueous gelatin solutions were studied. Gelatin is known to be a VoigtKelvin model system, in which elastic and viscous forces are parallel. In particular, we studied the gelation dynamics by repetitive measurements of the complex ACS spectrum. Different approaches to derive viscosity and shear modulus are applied and compared. In order to identify magnetoviscous effects in dynamic magnetic measurements, the magnetic field dependence of the Brownian relaxation time has to be eliminated. ACS measurements on various sufficiently strongly diluted aqueous MNP suspensions were performed in dependence of ac field amplitude and superimposed dc field strength and compared to theory. Excellent agreement was found.
\end{abstract}

Keywords: Brownian relaxation, Debye model, dynamic magnetic measurements, magnetic nanoparticles, Newtonian fluids, viscoelastic matrix

\footnotetext{
*Corresponding author: Frank Ludwig, Institut für Elektrische Messtechnik und Grundlagen der Elektrotechnik, TU Braunschweig, D-38106 Braunschweig, Germany, E-mail: f.ludwig@tu-bs.de Hilke Remmer, Institut für Elektrische Messtechnik und Grundlagen der Elektrotechnik, TU Braunschweig, D-38106 Braunschweig, Germany
}

Ә Open Access. ( 2020 Frank Ludwig and Hilke Remmer, published by De Gruyter. (c) BY-NC-ND This work is licensed under the Creative Commons Attribution-NonCommercial-NoDerivatives 4.0 International License. https://doi.org/10.1515/psr-2019-0115 


\section{Introduction}

The dynamics of magnetic nanoparticles (MNPs) have been widely studied theoretically [1-4] as well as experimentally [5-7]. It is characterized by two distinct mechanisms-the Brownian and the Néel one. In the former, the whole particle including shell rotates, and the Brownian relaxation time is given by

$$
\tau_{B}=\frac{3 \eta V_{h}}{k_{B} T}
$$

with hydrodynamic volume $V_{h}$, dynamic viscosity of the medium $\eta$, temperature $T$, and Boltzmann constant $k_{B}$. In the latter one, the magnetic moment flips thermally activated between easy axes, and the Néel relaxation time-for uniaxial anisotropy-is given by [2]

$$
\tau_{N}=\frac{\sqrt{\pi} \tau_{N 0}}{2\left(K V_{c} / k_{B} T\right)^{1 / 2}} \exp \left(\frac{K V_{c}}{k_{B} T}\right)
$$

Here $V_{c}$ is the (magnetic) core volume, $K$ is the effective anisotropy constant (or often denoted as effective anisotropy energy density) and $t_{\mathrm{NO}}$ is a constant of the order of $10^{-9} \mathrm{~s}$ [5]. For simplicity, Equation (2) is often simplified to

$$
\tau_{N}=\tau_{0} \exp \left(\frac{K V_{c}}{k_{B} T}\right) .
$$

If both processes can take place-for example when MNPs are suspended in a liquiddynamics in the small field limit is characterized by an effective relaxation time [8]

$$
\tau_{e f f}=\frac{\tau_{B} \tau_{N}}{\tau_{B}+\tau_{N}}
$$

i.e., the faster of the two mechanisms dominates. Figure 1 depicts the relaxation times for typical parameters of magnetite $\left(\mathrm{Fe}_{3} \mathrm{O}_{4}\right)$ MNPs in dependence of hydrodynamic diameter (spherical shapes are assumed, i.e., $V_{h}=(\pi / 6) d_{h}^{3}$ ) for different values of the dynamic viscosity.

Dynamic magnetic measurements combined with Equations (1)-(4) have been widely used for the characterization of MNPs [6,9-11]. While the Néel process contains information on the core size (to be more precise on the anisotropy energy $K V_{c}$ ), the Brownian relaxation times allows one to determine the hydrodynamic size of the MNPs, provided that the viscosity $\eta$ is known.

If the Brownian relaxation time is smaller than the Néel one, i.e., MNPs is thermally blocked, dynamic magnetic measurements have also been proposed for the realization of homogeneous bioassays [12-19]. Here the basic idea is that the hydrodynamic size and consequently the Brownian relaxation time increase upon specific binding of analytes to the functionalized surface of the MNPs. 


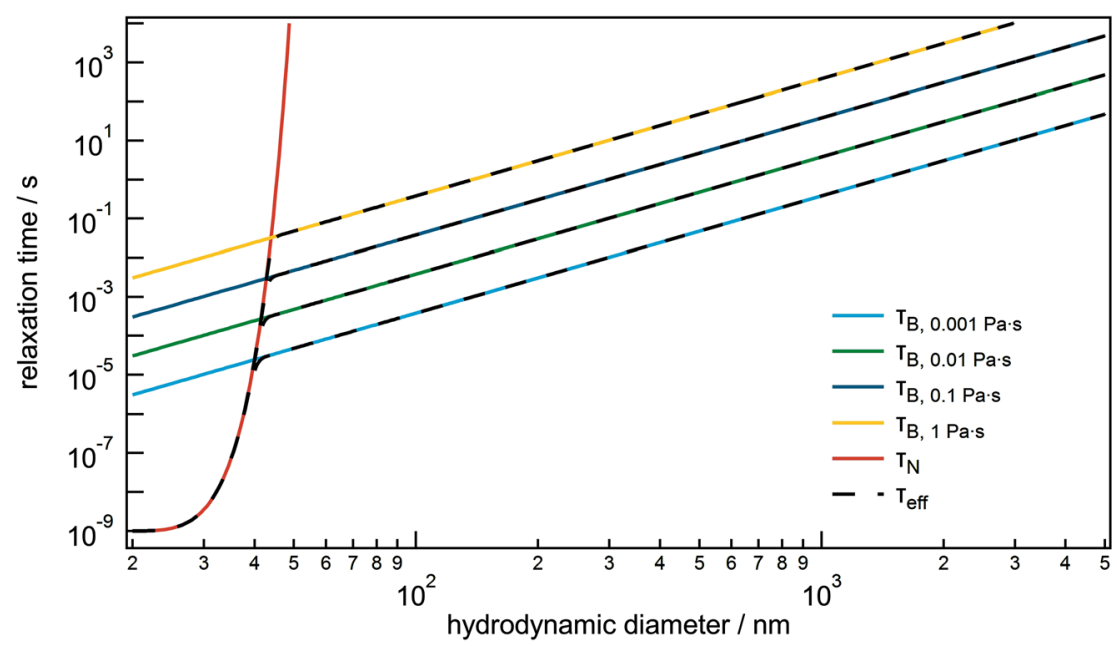

Figure 1: Relaxation time versus hydrodynamic diameter calculated for four different viscosities. Parameters: $K=10^{4} \mathrm{~J} / \mathrm{m}^{3}, T=296 \mathrm{~K}$, shell thickness $d_{s h}=10 \mathrm{~nm}, \tau_{0}=1 \mathrm{~ns}$.

On the other hand, if the MNPs are thermally blocked (the magnetic moment is "pinned" to the easy axes) and if their hydrodynamic size is known, dynamic magnetic measurements can be applied for the determination of the (local) dynamic viscosity. The use of MNPs as probes for measuring the local viscosity was first reported by Bacri et al. [20]. Later on, similar studies, utilizing different magnetic markers and different experimental techniques, were presented by a number of groups extending the work to non-Newtonian media [21-25]. In contrast to conventional rheology, applying MNPs as probes, the local environment is sensed on the nanoscale, yielding to the term "nanorheology". Nanorheological investigations can also be applied to understand the embedding of MNPs in the matrix and thus the particle-matrix interaction, which is central topic of the SPP1681 and this book. Depending on the characteristic length scales of the matrix and the size of the MNPs, rheological parameters, such as viscosity and shear modulus, derived from nanorheological measurements, can differ from the values obtained from standard rheology.

In this contribution, the basics of nanorheology based on MNPs as local probes are summarized. In particular, experimental techniques will be described and compared, and models for the estimation of (nano)rheological parameters from the experimental data will be discussed. The application of nanorheology will be described for two model systems: First, the dynamics of MNPs in water-glycerol mixtures, known to be Newtonian media, will be presented. Then, the gelation kinetics of aqueous gelatin solutions and the analysis of experimental data with the Voigt-Kelvin model will be presented. 
Regarding the control or material parameters by external magnetic fields, several groups reported on magnetoviscous effects applying conventional rheology [26]. Here one has to distinguish between the rotational viscosity, which is the hindrance of the rotation of single particles and which was theoretically described by Shliomis [27], and the increase of viscosity with rising magnetic field strength caused by dipolar interactions and thus structure formation [28]. While the former mechanism does-for geometric reasons-not take place in a dynamic magnetic measurement with stationary sample, the latter can principally occur. In order to identify magnetoviscous effects in nanorheology, one must, however, keep in mind that the Brownian relaxation time itself depends on the amplitude of the applied magnetic field. Therefore, the field dependence of the Brownian relaxation time is studied applying ac susceptometry and results are compared with theoretical models. It will be shown that excellent agreement with a theoretical model, based on solving the Fokker-Planck equation, is observed for Brownian relaxation.

\section{Dynamic magnetic measurement techniques}

In order to measure the dynamics of MNPs, several magnetic measurement techniques have been developed and established. Measurements can be carried out in the time or in the frequency domain. In time domain, one generally applies a magnetic field pulse, and measures the decay of the magnetic signal form the sample after switching off the field. This technique, also known as magnetorelaxometry (MRX), has been realized with various magnetic sensors. The pioneering MRX work at the PTB was realized using highly sensitive Nb SQUIDs as sensors $[6,29,30]$. In our work, we employ a custombuilt MRX setup based on fluxgate magnetometers. Although not reaching the resolution of the mentioned SQUID MRX setup, the differential fluxgate setup provides a number of practical advantages $[7,11]$.

In the frequency domain, most commonly ac susceptometry (ACS) is used. A sinusoidal magnetic field with amplitude in the range of a few $100 \mu \mathrm{T}$ is applied and a frequency sweep (at ideally constant field amplitude) is performed. The magnetic signal from the MNP sample is generally detected by a gradiometric detection coil. Many of the custom-built and the commercial ACS systems from Rise-Acreo [31] are designed for isothermal measurement around room temperature. Temperaturedependent measurements of the complex (ac) susceptibility $(\chi(T))$ are mainly performed utilizing commercial systems, such as the Magnetic Property Measurement System MPMS from Quantum Design [32]. The custom-built ACS systems applied in this work cover frequency ranges from $10 \mathrm{~Hz}$ to $10 \mathrm{kHz}$ (NF system with field amplitude of $567 \mu \mathrm{T}$ ) and $200 \mathrm{~Hz}-1 \mathrm{MHz}$ (HF system with field amplitude of $90 \mu \mathrm{T}$ ). Another system operating in the frequency domain is the fluxgate-based setup, which was originally realized for measurements of the response of the sample's magnetic moment to a rotating magnetic field [33]. The rotating magnetic field is generated by two Helmholtz 
coils. Frequency can be varied between 2 and $9 \mathrm{kHz}$. Using just one of the two Helmholtz coils, the system can also be operated as an ac susceptometer. In contrast to the ACS systems mentioned above, this system allows one to apply ac magnetic fields with amplitudes up to $9 \mathrm{mT}$. Alternatively, a small ac field can be superimposed by static magnetic fields having magnitudes of up to $9 \mathrm{mT}$ with directions parallel or perpendicular to the ac field. Thus, ACS measurements can also be carried out in the nonlinear regime, which allows one to study, e.g., the magnetic field dependence of relaxation times. In addition, this system allows a variation of sample temperature from room temperature to about $80^{\circ} \mathrm{C}$.

\section{Models for analysis of dynamic magnetic measurements}

\subsection{AC susceptibility of MNPs in Newtonian fluids}

The complex (ac) susceptibility is generally described by the Debye model and it is given by

$$
\chi(\omega)=\frac{\chi_{0}}{1+i \omega \tau_{e f f}} .
$$

Here $\omega$ is the angular frequency $2 \pi f$, and $\chi_{0}$ is the static susceptibility given by

$$
\chi_{0}=\frac{\mu_{0} n m^{2}}{3 k_{B} T}
$$

with vacuum permeability $\mu_{0}$, number density of magnetic nanoparticles $n$, and magnetic moment of a single MNP $m$. Splitting the complex susceptibility into real and imaginary parts, the following equations are obtained:

$$
\begin{gathered}
\chi^{\prime}(\omega)=\frac{\chi_{0}}{1+\left(\omega \tau_{\text {eff }}\right)^{2}} \\
\chi^{\prime \prime}(\omega)=\chi_{0} \frac{\omega \tau_{\text {eff }}}{1+\left(\omega \tau_{\text {eff }}\right)^{2}}
\end{gathered}
$$

Figure 2 shows real ( $\chi^{\prime}$ ) and imaginary part ( $\chi$ ”) versus the normalized frequency $\omega \tau_{\text {eff- }}$. Most pronounced is the maximum in the imaginary part, which lies at $\omega \tau_{\text {eff }}=1$. Consequently, from knowing the frequency of the maximum, the effective relaxation can be determined. In practice, there is a distribution of relaxation times $f\left(\tau_{\text {eff }}\right)$, so that Equation (5) modifies to

$$
\chi(\omega)=\chi_{0} \int_{0}^{\infty} \frac{f\left(\tau_{\text {eff }}\right)}{1+i \omega \tau_{e f f}} d \tau_{e f f}+\chi_{\infty}
$$




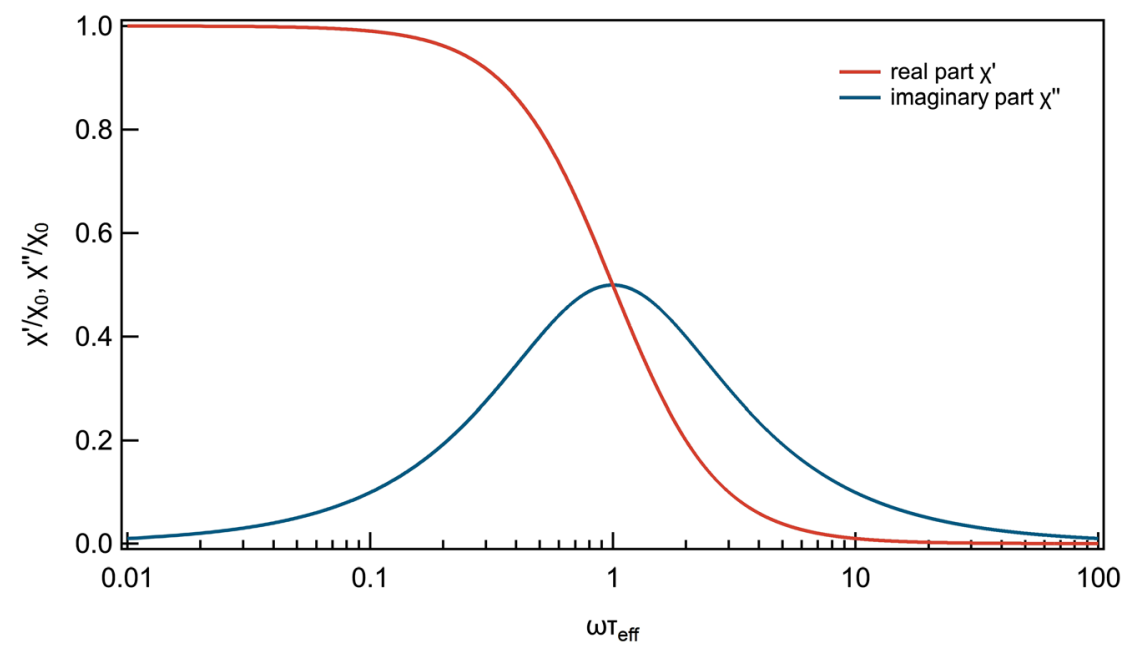

Figure 2: Real and imaginary parts of the ac susceptibility versus $\omega \tau_{\text {eff }}$ within the Debye model.

Here $\chi_{\infty}$ denotes the finite value of the real part at high frequencies, which is caused by either small superparamagnetic MNPs with relaxation times well below $1 \mu$ s and/or by intra-potential-well contributions [34-36].

Provided that the MNPs are thermally blocked, so that the effective relaxation time is given by the Brownian one, and assuming that the hydrodynamic size of the MNPs has some distribution with probability density function $f\left(V_{h}\right)$, Equation (9) modifies to

$$
\chi(\omega)=\chi_{0}^{* *} \int_{0}^{\infty} \frac{f\left(d_{h}\right)}{1+i \omega \tau_{B}} d d_{h}+\chi_{\infty}
$$

with

$$
\chi_{0}^{* *}=\frac{\mu_{0} n M_{s}^{2}}{3 k_{B} T}\left(\frac{\pi}{6} \bar{d}_{c}^{3}\right)^{2}
$$

Here $M_{s}$ is the saturation magnetization and $\bar{d}_{c}$ the mean core diameter (assuming spherical cores). If the MNPs are not fully blocked, i.e., the dynamics of a certain portion is dominated by the Néel mechanism, the following, more complex model has to be applied [37]:

$$
\chi(\omega)=\chi_{0}^{*} \int_{0}^{\infty} \int_{0}^{\infty} \frac{d_{c}^{6} f\left(V_{h}, V_{c}\right)}{1+i \omega \tau_{e f f}} d d_{c} d d_{h}+\chi_{\infty}
$$

with

$$
\chi_{0}^{*}=\frac{\mu_{0} n M_{s}^{2}}{3 k_{B} T}\left(\frac{\pi}{6}\right)^{2}
$$

Here $f\left(d_{c}, d_{h}\right)$ is a bivariate probability density function, which accounts for correlations between core and hydrodynamic size (for single-core MNP with very thin shell, the correlation factor between core and hydrodynamic size $\rho=1)$. For fitting ACS spectra measured on MNPs, generally a bimodal lognormal distribution is assumed: 
$f\left(d_{h}, d_{c}\right)=\frac{1}{2 \pi d_{c} d_{h} \sigma_{c} \sigma_{h} \sqrt{1-\rho^{2}}} \exp \left[-\frac{1}{2\left(1-\rho^{2}\right)}\left(\frac{\ln ^{2}\left(\frac{d_{c}}{\mu_{c}}\right)}{\sigma_{c}^{2}}+\frac{\ln ^{2}\left(\frac{d_{h}}{\mu h}\right)}{\sigma_{h}^{2}}-2 \rho \frac{\ln \left(\frac{d_{c}}{\mu_{c}}\right)}{\sigma_{c}} \frac{\ln \left(\frac{d_{h}}{\mu_{h}}\right)}{\sigma_{h}}\right)\right]$

These and various other generalizations of the Debye model for the determination of MNP structure parameters are described in detail in the study by Ludwig et al. [37].

\subsection{AC susceptibility of MNPs in non-Newtonian media}

Assuming again that the MNPs are thermally blocked so that only Brownian relaxation takes place, the Debye model can be modified to non-Newtonian media. Analogously to the model by DiMarzio and Bishop for the dielectric case [38], the spectrum of the complex susceptibility can be modified by replacing the dynamic viscosity $\eta$ by a complex value $\eta^{\star}=\eta^{\prime}+\mathrm{i} \eta^{\prime}$. With the complex shear modulus $G^{*}=G^{\prime}+\mathrm{i} G^{\prime \prime}=\mathrm{i} \omega \eta^{\star}$, Equation (5) reads

$$
\chi(\omega)=\frac{\chi_{0}}{1+A G^{*}}+\chi_{\infty}
$$

Here $A=\pi d_{h}^{3} /\left(2 k_{B} T\right)$ is a geometry factor, as introduced in the study by Roeben et al. [21]. Ignoring a distribution of hydrodynamic size, Equation (12)-normalized to $\chi_{0}$, corrected for $\chi_{\infty}$ and splitted into real and imaginary parts-can easily been used to determine both storage $G^{\prime}$ and loss modulus $G$ ' for each frequency [21]. The situation is more complicated if a distribution $f(A)$ must be considered. In the studies by Gratz and Tschöpe [39] and Sriviriyakula et al. [23], the distribution $f(A)$ is determined on a sample with known viscosity (generally using a Newtonian fluid) and assumed to remain unchanged when analyzing susceptibility spectra measured for nonNewtonian systems. A similar approach was proposed by Roeben et al. [21]. The proposed procedures turned out to work at least for non-Newtonian media with weak elastic contributions, such as PEG solutions [24].

The situation changes, if a certain viscoelastic model can be assumed. The simplest viscoelastic models are the Maxwell model with a viscous and an elastic term in series and the Voigt-Kelvin model with viscous and elastic terms in parallel. Basic rules for the arbitrary combination of viscous and elastic forces (torques) provide the following expressions for the complex shear modulus for the two cases:

$$
\begin{gathered}
G^{*}=\frac{i \omega \eta G}{G+i \omega \eta} \text { for the Maxwell model } \\
G^{*}=\eta+i \omega G \text { for the Voigt }- \text { Kelvin model }
\end{gathered}
$$

Here $\eta$ is the dynamic viscosity and $G=G^{\prime}$ is the storage part of the complex shear modulus. For example, for MNPs with a distribution of hydrodynamic diameters $f\left(d_{h}\right)$ 

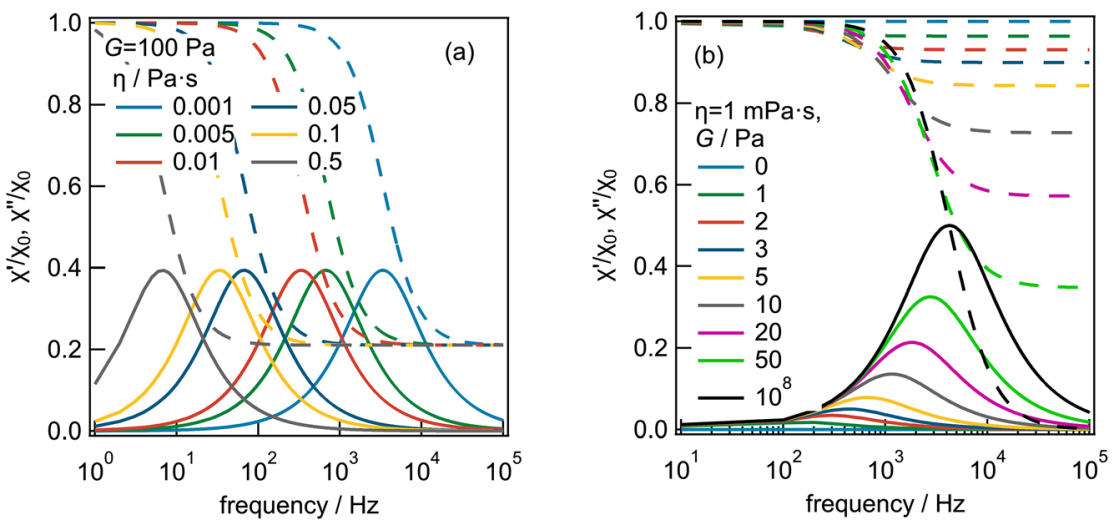

Figure 3: Simulated spectra for MNPs in a Maxwell-type medium. (a) Variation of viscosity and (b) variation of shear modulus $\left(d_{h}=46 \mathrm{~nm}, T=296 \mathrm{~K}\right)$.

embedded in a Voigt-Kelvin-like medium, the normalized complex susceptibility is given by

$$
\chi_{n}(\omega)=\frac{\chi(\omega)-\chi_{\infty}}{\chi_{0}^{* *}}=\int_{0}^{\infty} \frac{f\left(d_{h}\right)}{1+A G+i A \eta} d d_{h}
$$

The simulated susceptibility spectra for MNPs-ignoring a distribution $f\left(d_{h}\right)$-in a Maxwell-type medium are depicted in Figure 3. As for a Newtonian medium, an increase of the viscosity causes a shift of the susceptibility spectrum to lower frequencies (Figure 3(a)). An increase of the shear modulus $G$ causes an increase of the real part at high frequencies, while its zero-field value is not affected, and a shift of the position of the maximum in the imaginary part to higher frequencies, while its amplitude increases (Figure 3(b)). The limit of a Newtonian fluid is reached for $G \rightarrow \infty$.

Figure 4 depicts the simulated results for a Voigt-Kelvin model. As before, an increase of the viscosity causes a shift of the spectrum to lower frequencies. However, an increase of the shear modulus $G$ results in a decrease of the real part at low frequency and of the amplitude of the maximum of the imaginary part. In contrast to the Maxwell model, the real part of the susceptibility values at high frequencies decreases to zero, independent of shear modulus. An increase of $G$ causes a shift of the frequency at which the maximum in the imaginary part occurs to higher frequencies and a decrease of its amplitude. Note that this behavior is qualitatively similar to the case when a static magnetic field is superimposed [40].

The same general behavior for the complex susceptibility of a Maxwell and a VoigtKelvin system was also derived by Tschöpe et al. [22] by solving the regarding equations of motion. Importantly, information on what type of viscoelastic model one deals with 

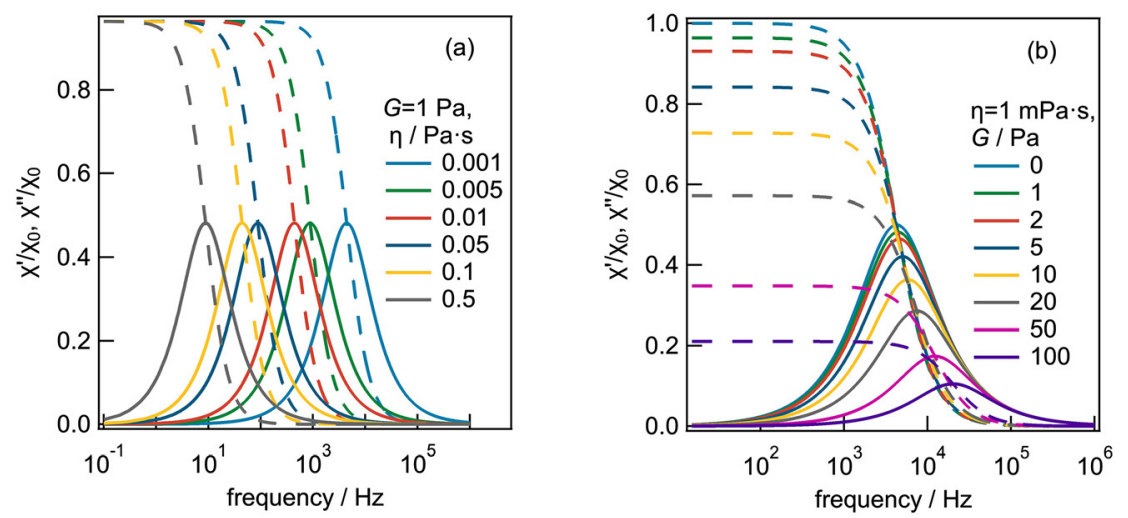

Figure 4: Simulated spectra for MNPs in a Voigt-Kelvin-type medium. (a) Variation of viscosity and (b) variation of shear modulus $\left(d_{h}=46 \mathrm{~nm}, T=296 \mathrm{~K}\right)$.

can be seen from the general characteristics of the complex spectrum, which significantly differs for a Maxwell and a Voigt-Kelvin system.

Theoretical models for the complex susceptibility of thermally blocked MNPs in Maxwell- and Voigt-Kelvin-type matrices were proposed by Raikher and colleagues [41-43]. Here the authors solved the regarding equations of motion including thermal agitation. In the following, we will focus on the Voigt-Kelvin model [41].

The equation of motion is given by

$$
I \ddot{\vartheta}+\zeta \dot{\vartheta}+K \vartheta=y(t)
$$

with the angle between magnetic moment and applied magnetic field $\vartheta$, the moment of inertia of the particle $I$, the rotational friction coefficient $\zeta=6 \eta V_{h}$, the linear elastic restoring parameter $K=6 G V_{h}$ and the stochastic driving torque due to thermal energy $y(t)$. The magnetic torque is assumed to be small. For the dynamic susceptibility, the following expressions were derived:

$$
\chi_{\alpha}(\omega)=\chi_{0, \alpha}\left(1+i \omega \int_{0}^{\infty} d \tau e^{i \omega \tau} G_{\alpha}(\tau)\right)
$$

with

$$
\chi_{0, \|}=\frac{n m^{2}}{k_{B} T} \exp \left(-\frac{k_{B} T}{K}\right)\left[\cosh \left(\frac{k_{B} T}{K}\right)-1\right] \text { and } \chi_{0, \perp}=\frac{n m^{2}}{k_{B} T} \exp \left(-\frac{k_{B} T}{K}\right) \sinh \left(\frac{k_{B} T}{K}\right)
$$

as well as

$$
G_{\|}(\tau)=\frac{\left(\cosh \left(\frac{k_{B} T}{K} \exp \left(-\frac{t}{\tau_{K}}\right)\right)-1\right) \exp (i \omega t)}{\cosh \left(\frac{k_{B} T}{K}\right)-1} \text { and } G_{\perp}(\tau)=\frac{\sinh \left(\frac{k_{B} T}{K} \exp \left(-\frac{t}{\tau_{K}}\right)\right) \exp (i \omega t)}{\sinh \left(\frac{k_{B} T}{K}\right)} .
$$



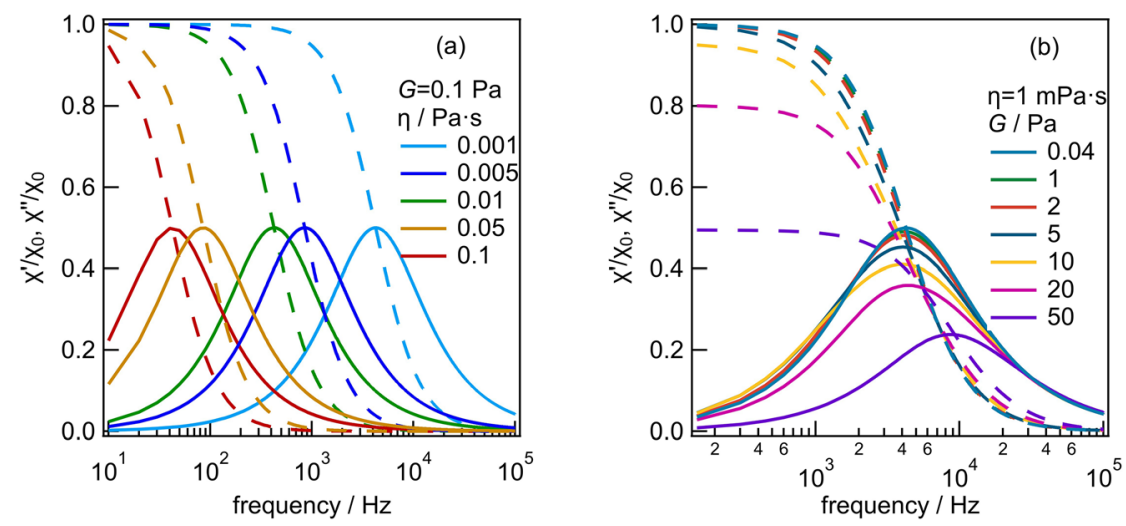

Figure 5: Spectra for MNPs in a Voigt-Kelvin-type medium calculated with model by Raikher et al. (a) Variation of viscosity and (b) variation of shear modulus $\left(d_{h}=46 \mathrm{~nm}, T=296 \mathrm{~K}\right)$.

The total susceptibility is then given by

$$
\chi_{\text {tot }}(\omega)=\frac{1}{3}\left(\chi_{\|}+2 \chi_{\perp}\right) .
$$

The symbol $\alpha$ in Equations (15) denotes the orientations parallel (II) or perpendicular $(\perp)$ to the excitation field. The time constant $\tau_{K}=\zeta / K$. Note that the equation for the Brownian relaxation time in the study by Raikher et al. [41] differs from the expression in Equation (1) by a factor of 2 (this also results in a shift of the frequency of the maximum in the imaginary part when solving Equations (15) and (16) in the limit of vanishing elasticity), so that we tentatively replaced $k_{B} T$ in Equation (15) by $2 k_{B} T$ $[25,44]$.

Lateron, Rusakov et al. [45] extended their theoretical work, which for the case described above is limited to the case of planar (1D) rotation, to complete angular space (2D). The equations for the complex susceptibility they obtained qualitatively differ, however, from the ones derived for the 1D case (Equations (15) and (16)) and from the experimental data, which will be described in Section 4.2. Therefore, spectra measured on blocked MNPs in a Voigt-Kelvin-type matrix were analyzed with Equations (15) and (16).

Figure 5 displays the ac susceptibility spectra numerically calculated with Equations (15) and (16) for the same set of parameters as for Figures 3 and 4. Qualitatively, the same behavior as in Figure 4 is obtained. In comparison with the modified Debye model (Equation (13)), the numerical model by Raikher et al. requires considerably higher computational effort, especially when extending it by parameter distributions and implementing it in fitting routines. 


\subsection{Magnetorelaxometry of MNPs in Newtonian fluids}

For a sample consisting of identical MNPs, an exponential decay of the relaxation signal is expected:

$$
B(t) \sim \exp \left(-\frac{t}{\tau_{e f f}}\right)
$$

If there are distributions of core and hydrodynamic size, generally the cluster magnetic moment superposition model (CMSM) - as originally proposed by Eberbeck et al. [6]-is applied. Here the decay of the net magnetic moment of the sample is given by $[6,11]$

$$
B(t)=\Phi M_{s} \int_{0}^{\infty} f\left(V_{h}\right) \int_{0}^{\infty} f\left(V_{c}\right) V_{c} L\left(V_{c}\right)\left[1-\exp \left(-\frac{t_{m a g}}{\tau_{e f f, H}}\right)\right] \exp \left(-\frac{t}{\tau_{e f f}}\right) d V_{c} d V_{h}
$$

with a system factor $\Phi$ and the Langevin function $\mathrm{L}\left(V_{c}\right)$. The Langevin function is given by $L(\xi)=\operatorname{coth}(\xi)-1 / \xi$ with the Langevin parameter $\xi=m B /\left(k_{B} T\right)$. Note that the effective relaxation time $t_{\text {eff, } H}$ comprises the Brownian and Néel relaxation times in a static magnetic field. If the MNPs are thermally blocked, $t_{\mathrm{eff}}$ and $t_{\mathrm{eff}, H}$ can be replaced by $t_{\mathrm{B}}$ and $t_{\mathrm{B}, \mathrm{H}}$, respectively, and integration has to be carried out over $V_{h}$ only.

\subsection{Magnetorelaxometry of MNPs in viscoelastic matrices}

Currently, there are no models, which describe the complete magnetorelaxation signal for a given type of viscoelasticity. In the studies by Raikher and coworkers [41, 45], an equation for the effective relaxation time of thermally blocked MNPs in a Voigt-Kelvintype matrix is provided:

$$
\tau_{e f f, V K}=\tau_{B}\left(1+\frac{K}{2 k_{B} T}\right)
$$

Here $t_{\mathrm{B}}$ is the Brownian relaxation time in a purely viscous medium (Equation (1)), and $K=6 G V_{h}$ is the elastic restoring parameter. Note that again a difference of a factor of 2 is found comparing the studies by Raikher et al. [41] and Rusakov and Raikher [45]. Equation (19) already includes the correction.

In order to analyze experimental MRX curves measured on MNPs in a Voigt-Kelvintype matrix, Equation (18) with expression (19) for the effective relaxation time can be applied.

\subsection{Magnetic field dependence of Brownian relaxation time}

Equations (1) and (2) provide the zero-field expressions for the Brownian and Néel relaxation times. In standard ACS measurements, where ac magnetic fields with 
amplitudes of well below $1 \mathrm{mT}$ are applied, i.e., the Langevin parameter $\xi=\mathrm{mB}$ / $\left(k_{B} T\right) \ll<1$, they can reliably be used in the analysis. However, as soon as $\xi$ becomes larger, significant changes of the relaxation time constants occur.

The dependence of the Brownian relaxation time of thermally blocked MNPs in large ac magnetic fields was theoretically studied by Yoshida and Enpuku [46] by solving the Fokker-Planck equation. Based on the simulated ACS spectra, they derived a set of empirical equations, which can be used to fit experimental data. Here the Brownian relaxation time in dependence of Langevin parameter $\xi$ is given by $[11,40]$

$$
\tau_{B, H}=\frac{\tau_{B}}{\sqrt{1+0.126 \xi^{1.72}}},
$$

i.e., the Brownian relaxation time decreases with increasing ac field amplitude. Similar expressions were also derived by Gratz and Tschöpe [47] as well as by Fock et al. [48].

The Brownian relaxation time for the case of a superimposed static magnetic field-either parallel or perpendicular to the ac probing field-were theoretically derived by Martsenyuk et al. [8] and are given by

$$
\tau_{\text {par }}=\frac{d \ln L(\xi)}{d \ln \xi} \tau_{\mathrm{B}} \text { and } \tau_{\text {perp }}=\frac{2 L(\xi)}{\xi-L(\xi)} \tau_{\mathrm{B}} .
$$

The situation is more difficult for the Néel relaxation since one has to account for the generally random distribution of easy axes. The Néel relaxation of MNPs with their easy axes parallel to an applied static magnetic field is given by [2]

$$
\tau_{N, H}=\frac{\sqrt{\pi} \tau_{N 0}}{\sigma^{3 / 2}\left(1-h^{2}\right)}\left[(1+h) \exp \left(-\sigma(1+h)^{2}\right)+(1-h) \exp \left(-\sigma(1-h)^{2}\right)\right]^{-1}
$$

with the normalized magnetic field $h=B / B_{\mathrm{k}}, B_{\mathrm{k}}=2 K / M_{\mathrm{s}}$ and $\sigma=K V_{\mathrm{c}} /\left(k_{\mathrm{B}} T\right)$. There is no expression for the Néel relaxation time of an ensemble of randomly oriented MNPs in a large ac magnetic field.

\section{Experimental results}

\subsection{ACS and MRX measurements on MNPs in Newtonian media}

ACS and MRX measurements were performed on a series of samples with different viscosities. Here water-glycerol mixtures were prepared. First, SHP-25 from Ocean Nanotechnology was studied. These MNPs are single core, thermally blocked nanoparticles consisting of magnetite and having a nominal (geometric) core diameter of $25 \mathrm{~nm}$ with a narrow size distribution [40]. Figure 6 depicts the spectra of the imaginary part of the complex susceptibility for samples with glycerol contents ranging from 0 to $86.8 \mathrm{wt} \%$. One discerns a pronounced maximum, which shifts with increasing viscosity to lower frequencies, as expected for Brownian-dominated MNPs. In contrast to the 


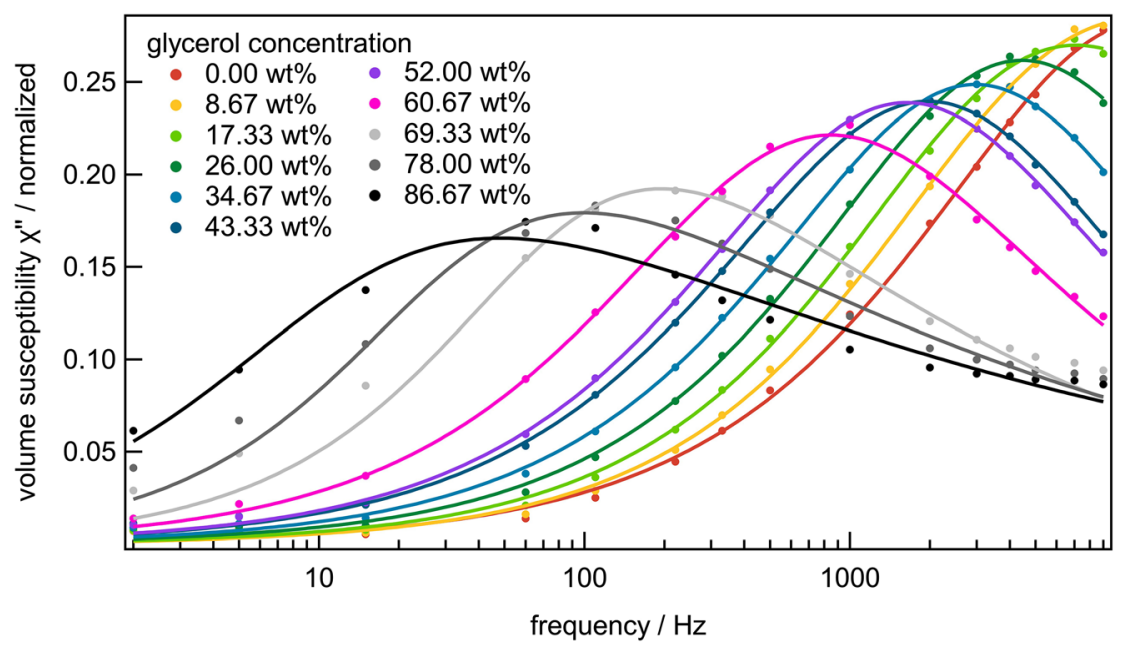

Figure 6: Imaginary part versus frequency for SHP-25 MNPs suspended in different water-glycerol mixtures. Numbers in inset give glycerol concentration. Symbols are measured data points, lines are fits with phenomenological Havriliak-Negami model (Equation (23)).

theoretical expectations (cf. Figures 4(a) and 5(a)), its amplitude decreases, which is compensated by an increasing width of the imaginary part peak, which is related to an increase of the distribution of Brownian relaxation times.

In order to precisely determine the frequency $f_{c h}$ at which the imaginary part peaks, data points were fitted with the phenomenological Havriliak-Negami model. Here the complex susceptibility is given by

$$
\chi=\frac{\chi_{a m p}}{\left(1+(i \omega \tau)^{1-\alpha}\right)^{\beta}}+\chi_{\infty}
$$

where $\chi_{\infty}$ is the real-valued susceptibility at high frequencies, and $\chi_{\mathrm{amp}}$ is the amplitude of the frequency-dependent part. The parameter $\alpha$ accounts for the width of the spectrum, while the parameter $\beta$ reflects its asymmetry. For $\alpha=0$ and $\beta=1$, the Debye model is reconstituted.

The values for the dynamic viscosity were estimated from the measured imaginary part applying

$$
\eta=\frac{k_{B} T}{6 \pi f_{c h} V_{h}}
$$

which is obtained from Equation (1) and the condition $2 \pi f_{\mathrm{ch}} \tau_{B}=1$.

To determine the viscosity values from the MRX curves depicted in Figure 7, Equation (18) was applied. The parameters of core diameter distribution and of the anisotropy energy density were estimated from measurements on an immobilized (by freeze-drying in a mannitol matrix) reference sample. Knowing these parameters, the 


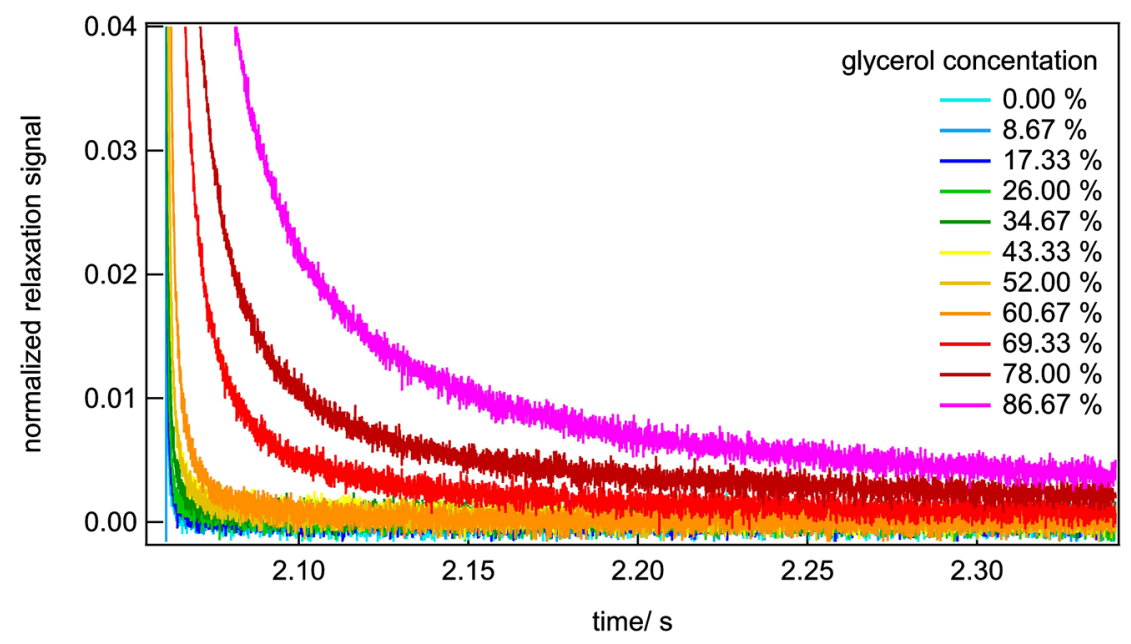

Figure 7: Normalized relaxation curves measured on SHP-25 MNPs suspended in different waterglycerol mixtures.

parameters of the distribution of hydrodynamic diameter were obtained from the MRX curve measured on an aqueous suspension, i.e., with known viscosity. Then keeping these parameters constant, the viscosity is the only free parameter for fitting the relaxation curves for SHP-25 MNPs in water-glycerol mixtures.

The viscosity values, estimated from ACS and MRX data, are displayed in Figure 8 versus the glycerol concentration. Good agreement with the theoretical values calculated with the equation by Cheng [49] is found.

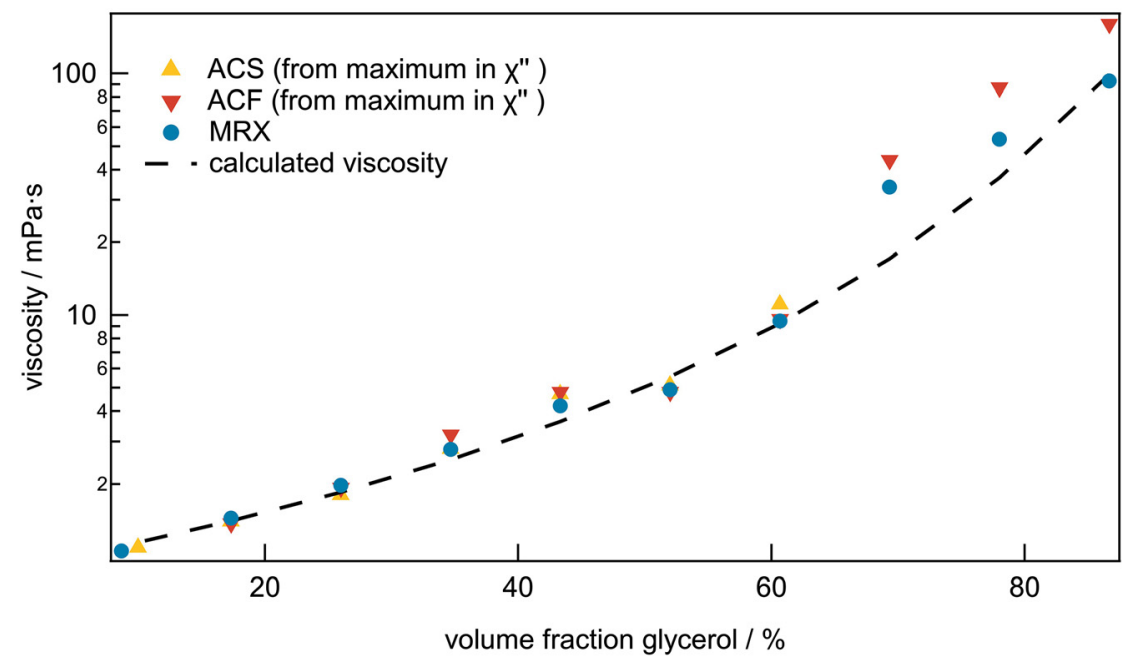

Figure 8: Viscosity values determined from ACS, ACF (refers to fluxgate-based ACS setup) and MRX measurements on SHP-25 versus concentration of glycerol. Dashed line displays theoretical viscosity calculated using the equation by Cheng [49]. 


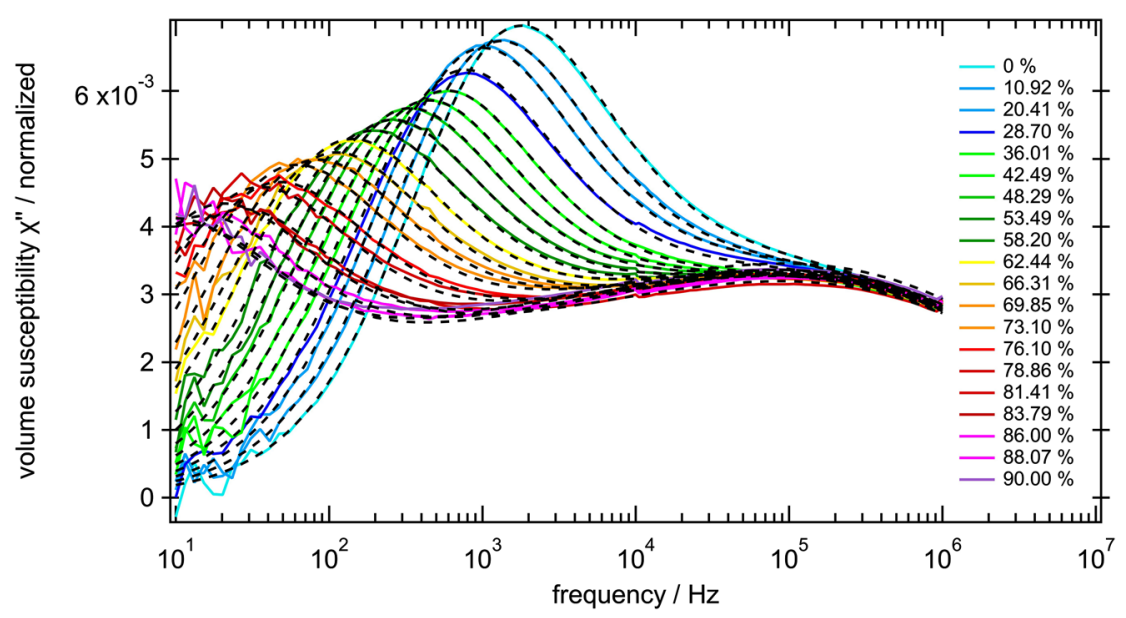

Figure 9: Imaginary part versus frequency for FeraSpin XL MNPs suspended in different waterglycerol mixtures. Numbers in inset give glycerol concentration. Symbols are measured data points, lines are fits with Equation (11).

In order to test, how reliably dynamic viscosities can be determined from ACS measurements on MNP systems, which exhibit Brownian and Néel contributions, measurements on a viscosity series of FeraSpin XL from nanoPET Pharma GmbH were performed. FeraSpin XL is a size fraction of FeraSpin $\mathrm{R}$ with a mean hydrodynamic diameter of about $60 \mathrm{~nm}$. FeraSpin $\mathrm{R}$ is a multi-core particle system comprising of densely packed iron oxide cores with sizes of 5-7 $\mathrm{nm}$ [50]. The imaginary parts of the measured ACS spectra are shown in Figure 9. The aqueous sample measurement displays a maximum at about $1.8 \mathrm{kHz}$ and a shoulder around $100 \mathrm{kHz}$. With increasing viscosity, the frequency of the low-frequency maximum shifts to lower frequencies, indicating that it is caused by Brownian-dominated MNPs while the wide highfrequency maximum remains unchanged, i.e., it is related to Néel relaxation.

To fit the measured spectra, Equation (11) is applied. For simplicity, the correlation coefficient $\rho$ is set to zero (i.e., we ignore a correlation between core and hydrodynamic size). As for the analysis of the MRX curves in Figure 7, core parameters are independently estimated from relaxation curves of the immobilized reference samples, while the parameters of the hydrodynamic size distribution were obtained from the analysis of the ACS spectrum measured on the aqueous suspension of FeraSpin XL. Dashed lines in Figure 9 display the fitted spectra. Note that-for all samples except the aqueous one-viscosity is the only free parameter. In contrast to the viscosity series measured on SHP-25, the standard deviation of the lognormal distribution $\sigma_{h}$ of hydrodynamic diameters (and Brownian relaxation times) remained nearly constant ( $\sigma_{h} \approx 0.28$ ). Consequently, the observed decay in the amplitude of the maximum is caused by a gradual decrease of the Brownian-dominated MNP portion with increasing 


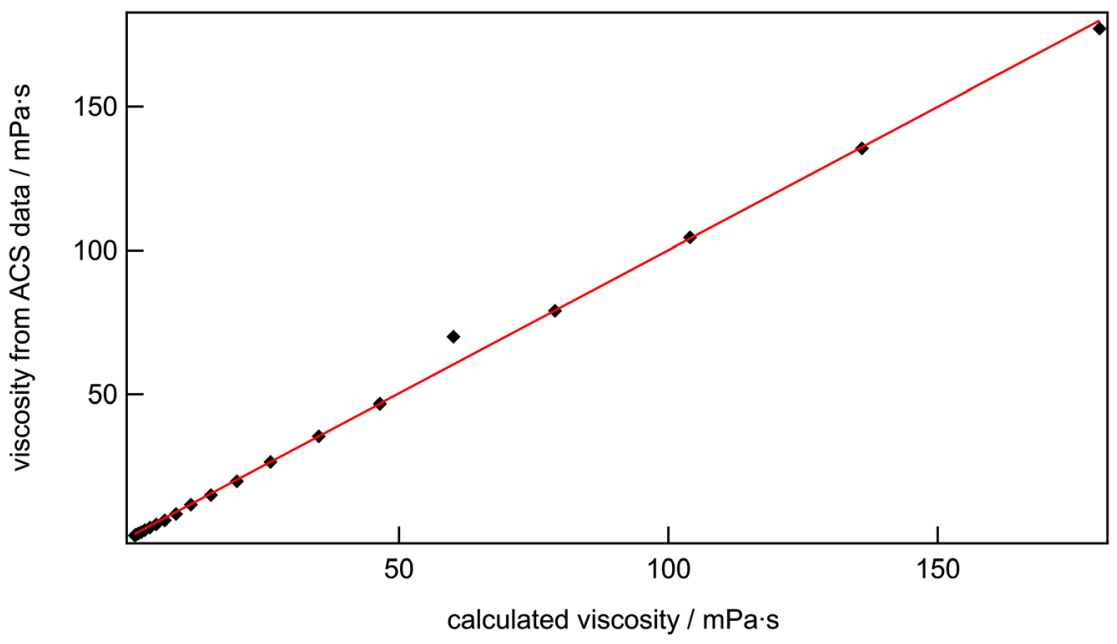

Figure 10: Dynamic viscosities determined from ACS spectra on FeraSpin XL viscosity series versus calculated values.

viscosity (transition from Brownian to Néel relaxation). As Figure 10 shows, excellent agreement between experimental and theoretical values is found again.

\subsection{Investigation of gelation dynamic of aqueous gelatin suspensions}

As Voigt-Kelvin-type matrix system, aqueous gelatin solutions are chosen. As demonstrated by Tschöpe et al. [22] using Ni nanorods as magnetic markers, rheological parameters of aqueous gelatin solutions from dynamic opto-magnetic measurements can well be estimated on the basis of the Voigt-Kelvin model. Here single-core $\mathrm{CoFe}_{2} \mathrm{O}_{4}$ synthesized at the University of Cologne [21] are applied as markers. They are surrounded by a PAA shell, have mean core diameters of $15 \mathrm{~nm}$ and a hydrodynamic diameter-when suspended in DI water-of $18 \mathrm{~nm}$. Due to the comparably high anisotropy energy density, $\mathrm{CoFe}_{2} \mathrm{O}_{4}$ nanoparticles of this size are well thermally blocked.

Similarly to the procedure executed by Tschöpe et al. [22], $\mathrm{CoFe}_{2} \mathrm{O}_{4} \mathrm{MNPs}$ were suspended in aqueous gelatin solutions with different gelatin contents $(2.5,5,7.5$ and $10 \mathrm{wt} \%$ ). These suspensions were first heated up to $40^{\circ} \mathrm{C}$, thus being in the sol state, and the hydrodynamic diameter was determined from the position of the maximum in the ACS imaginary part and the known viscosity at $40{ }^{\circ} \mathrm{C}$. The viscosity values at $40{ }^{\circ} \mathrm{C}$ were independently determined using an Anton Paar SVM 3000 Stabinger viscosimeter. Knowing the dynamic viscosity at this temperature, the ACS spectra measured at $40{ }^{\circ} \mathrm{C}$ were fitted with the Debye model extended by inserting a lognormal distribution of hydrodynamic diameters. For the $2.5 \mathrm{wt} \%$ sample, a mean hydrodynamic diameter of 


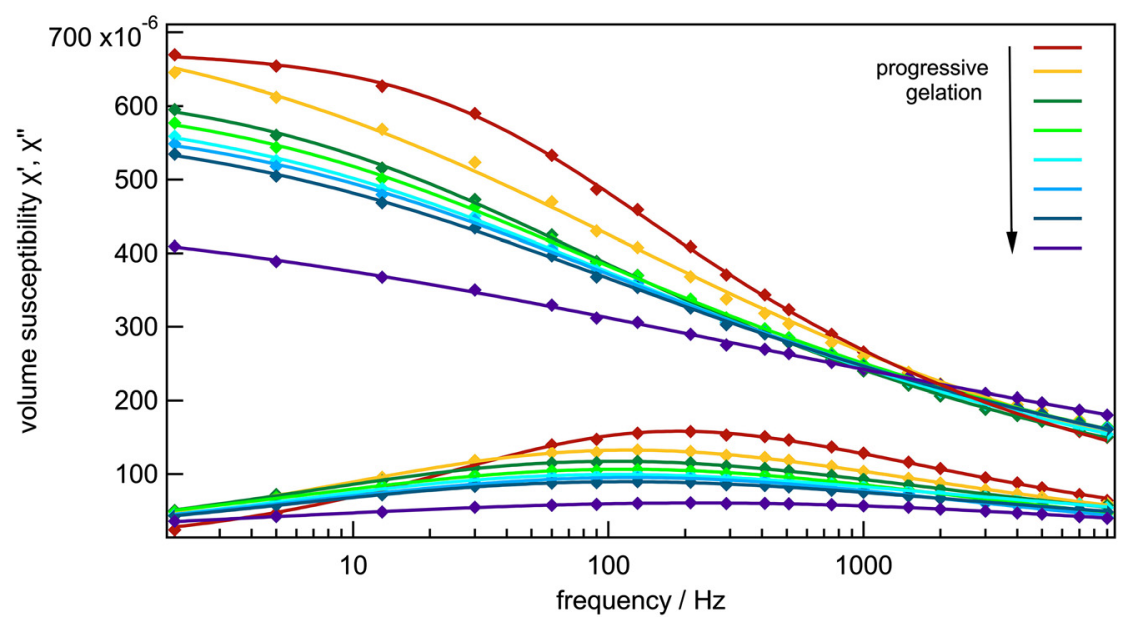

Figure 11: Real and imaginary parts of ACS spectra recorded at different stages of the gelation process.

$37 \mathrm{~nm}$ was determined; for the $5 \mathrm{wt} \%$ sample, a value of $46 \mathrm{~nm}$ was found. The increase in hydrodynamic size compared to the value of the aqueous sample at room temperature is attributed to an adsorbent gelatin layer. Repeated heating of the samples to $40^{\circ} \mathrm{C}$ after gelation always resulted in the same hydrodynamic size, so that we assume that the thickness of the absorbent gelatin layer does not change with time.

Figure 11 depicts real and imaginary part of the sample with $5 \mathrm{wt} \%$ gelatin, measured at different stages of the gelation process. Apparently, the magnitude of the real part at low frequencies continuously decreases with increasing gelation time while it approaches zero at high frequencies, as expected for a Voigt-Kelvin-type system.

Figure 12(a) and (b) shows the ACS imaginary parts measured on the (a) 2.5 and (b) $5 \mathrm{wt} \%$ samples. For the $2.5 \mathrm{wt} \%$ gelatin sample, a gradual shift of the peak frequency to
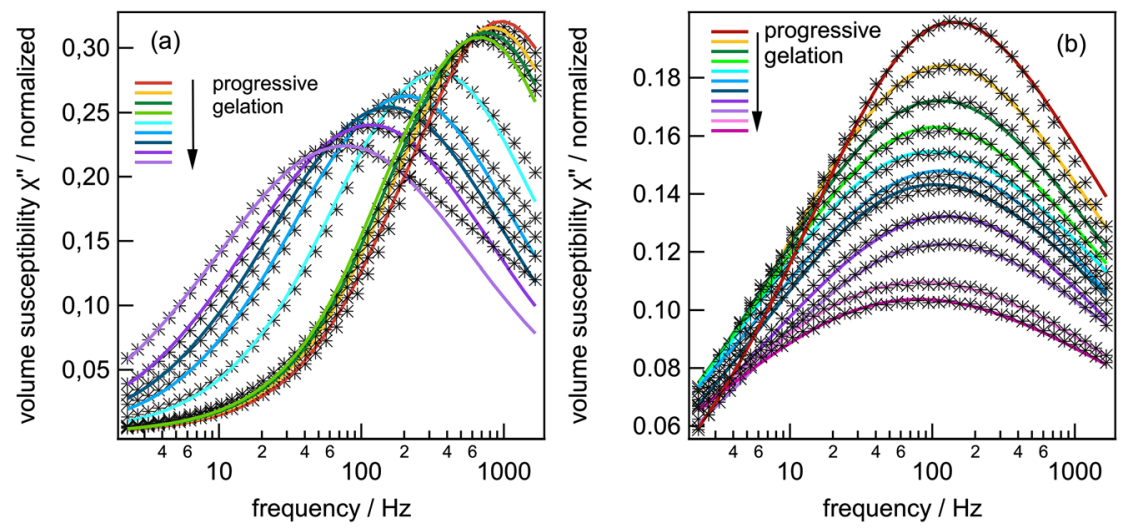

Figure 12: Evolution of ACS imaginary part of (a) sample with 2.5 wt $\%$ gelation and (b) with 5 wt $\%$ gelatin. Symbols show data points, lines are fits with Equations (15) and (16). 
lower frequencies and of its magnitude is observed. At the same time, the width of the spectrum increases with increasing gelation time. In contrast, the spectrum of the imaginary part measured on the $5 \mathrm{wt} \%$ gelatin sample first shows a shift of the peak frequency to lower frequencies but after a certain time, it reverses and increases again. But similarly to the $2.5 \mathrm{wt} \%$ sample case, the magnitude of the maximum decreases and the width increases with increasing gelation time.

The behavior observed for the $5 \mathrm{wt} \%$ sample is qualitatively similar to that observed by Tschöpe et al. [22] applying an oscillating magnetic field to Ni nanorods and detecting their response by a magneto-optic technique.

Reminding the fundamentals of a Voigt-Kelvin model (Section 3.2), the observed behavior is very clear: An increase of the (local) dynamic viscosity of the medium causes a shift of the imaginary part to lower frequencies. An increase of the shear modulus results in a decrease of the ACS magnitude and a shift of the position of the $\chi$ " maximum to higher frequencies. The latter effect is very pronounced for the sample with $5 \mathrm{wt} \%$ gelatin. But at the same time, a significant increase of the width of the spectrum is discernable. In order to extract values for viscosity $\eta$ and shear modulus $G$, the measured spectra of the imaginary part were fitted with Equations (15) and (16). Since they do not consider distributions of parameters, they were generalized by inserting lognormal distributions of hydrodynamic diameter $f\left(d_{h}\right)$ and (local) dynamic viscosity $f(\eta)$. Obtained parameters for $\eta$ and $G$ are shown in Figure 13 as a function of gelation time.

Obviously, the same general trend is found for both samples. The viscosity continuously increases with rising gelation time, while the shear modulus first increases, followed by a plateau and then starts rising again. The main difference between both samples lies in the different magnitudes of the (nano)rheological parameters. While viscosity and shear modulus of the $2.5 \mathrm{wt} \%$ sample at $1000 \mathrm{~min}$ amount to about $10 \mathrm{mPa}$ s and $8 \mathrm{~Pa}$, respectively, they are $30 \mathrm{mPa}$ s and $9 \mathrm{~Pa}$ for the $5 \mathrm{wt}$
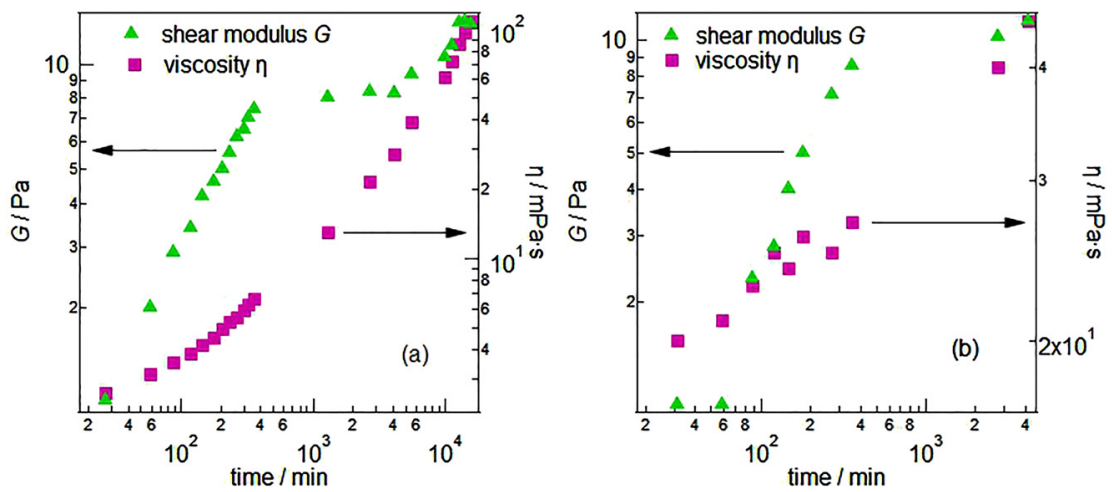

Figure 13: Viscosity $\eta$ and shear modulus $G$ as a function of gelation time for the samples with (a) 2.5 and (b) 5 wt $\%$ gelatin. 
$\%$ sample. The different ratio of viscosity and shear modulus causes that the elastic term starts dominating for the $5 \mathrm{wt} \%$ gelatin sample at high gelation times.

Similar findings were obtained when analyzing the measured ACS spectra with the modified Debye model (Equation (13) extended by a distribution of local viscosities). Figure 14 displays the fitting results obtained with the numerical model by Raikher et al. [41] and the modified Debye model. The viscosities estimated by both approaches agree very well. Regarding shear modulus, its evolution with gelation time is qualitatively the same but the absolute values differ by a factor of about two. At this stage, it remains open what the reason is. As pointed out in Section 3.2, $k_{B} T$ in the original equations given in the paper by Raikher et al. [41] was replaced by $2 k_{B} T$ so that - in the limiting case of vanishing elasticity-the well-known expressions for the Brownian relaxation time and the standard Debye model are re-established.

According to Normand et al. [51], there are four phases in the gelation kinetics of gelatin. Comparing Figure 13 with their findings, the time interval up to $300 \mathrm{~min}$ can be attributed to phase 2, which is characterized by the gel formation and a rapid increase of $G$ and $\eta$. The time up to about 4000 min may be related to phase 3, which-according to Normand et al.-is characterized by the extension of existing cross-links in the network rather than the formation of new ones. The rise of $G$ at longer times-as discernable in Figures 13 and 14-was also observed by the authors applying a stresscontrolled rheometer technique.

The ACS spectra measured on samples with 7.5 and $10 \mathrm{wt} \%$ gelatin could not be analyzed since the characteristic features were outside the accessible frequency window.

The same samples were also investigated by magnetorelaxometry. The aqueous gelatin solution samples were heated to $40{ }^{\circ} \mathrm{C}$ for about $60 \mathrm{~min}$, and after quickly

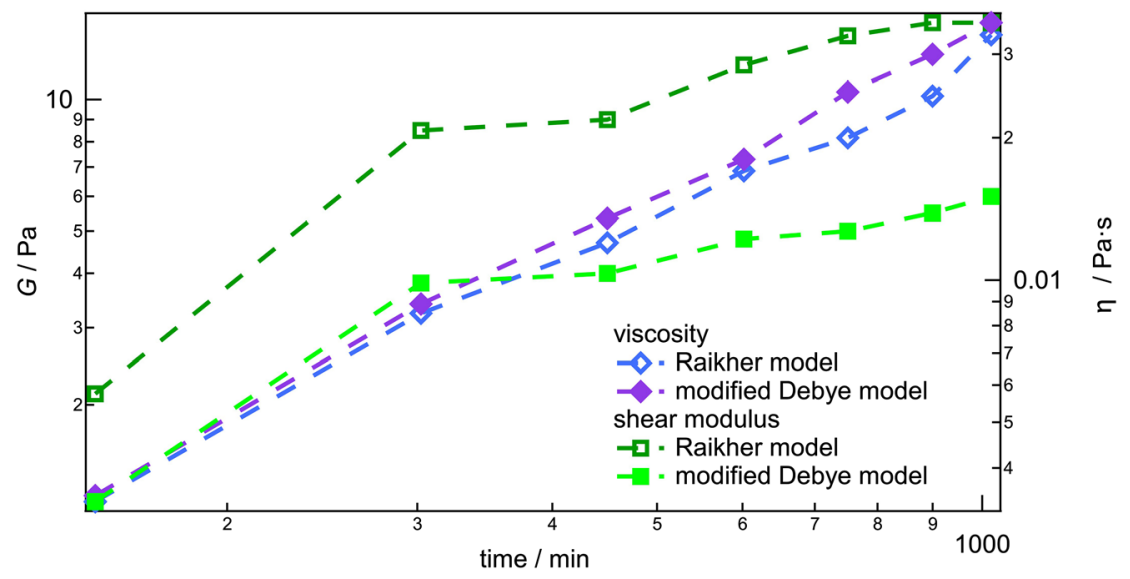

Figure 14: Comparison of temporal evolution of viscosity and shear modulus values determined from measured ACS spectra with numerical model by Raikher et al. [41] and modified Debye model. 

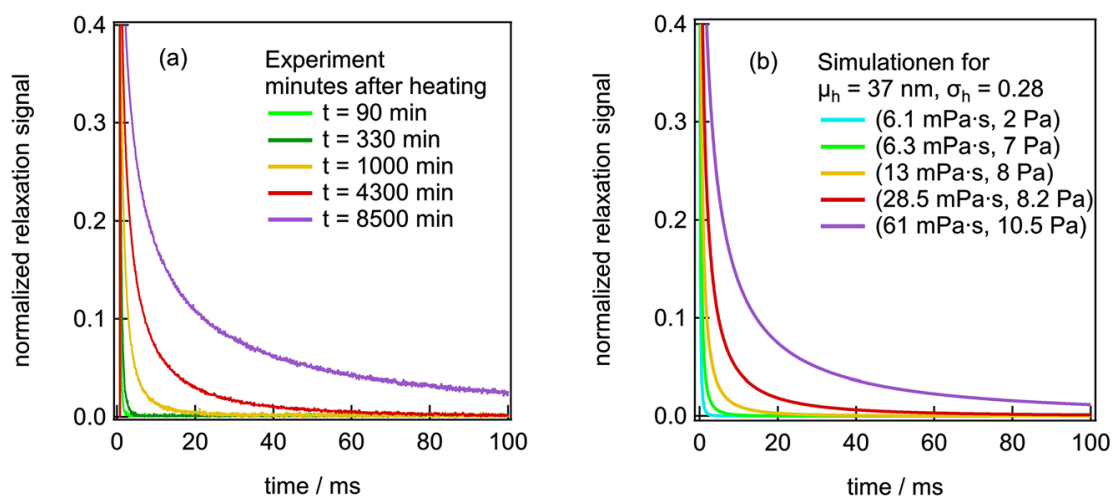

Figure 15: Normalized (a) measured and (b) simulated MRX curves of the samples with 2.5 wt $\%$ gelatin. The numbers in brackets in the legend of (b) denote the applied values for viscosity and shear modulus.

cooling them down to $23{ }^{\circ} \mathrm{C}$, successive MRX measurements were performed. The measured MRX curves, measured on the $2.5 \mathrm{wt} \%$ gelatin sample and normalized to the signal before the magnetic field pulse is switched off, are depicted in Figure 15(a). With increasing gelation time, the decay of the relaxation signal monotonously slows down. An independent estimation of viscosity $\eta$ and shear modulus $G$ by fitting the measured MRX curves with Equations (18) and (19) is not possible. Therefore, Figure 15(b) shows the normalized MRX curves simulated with Equations (18) and (19) using the parameters obtained from the analysis of the corresponding ACS spectra. A reasonable agreement is found. A qualitatively similar behavior was observed for the $5 \mathrm{wt} \%$ gelatin sample. The behavior measured for the 7.5 and $10 \mathrm{wt} \%$ gelatin samples is more complex. The not normalized MRX curves for the $7.5 \mathrm{wt} \%$ gelatin sample are depicted in Figure 16(a). The change of the curves with increasing gelation is no longer monotonous and even a crossing can be observed. As for the ACS case, this may be caused by the different effect of viscosity and shear modulus changes on the MNP dynamics. Figure 16(b) shows the MRX curves measured on the sample with $10 \mathrm{wt} \%$ gelatins at different times after reaching $23{ }^{\circ} \mathrm{C}$. Apparently, no significant differences between curves and the reference curve measured on a freeze-dried sample of $\mathrm{CoFe}_{2} \mathrm{O}_{4}$ nanoparticles are discernable, meaning that the dynamics of this sample is dominated by the Néel mechanism.

\subsection{Investigation of magnetic field dependence of Brownian relaxation time}

All ACS measurements described in Section 4.1 and 4.2 were carried out at small ac field amplitudes $(200 \mu$ T, i.e., $\xi \ll 1)$. To extract nanorheological parameters from 

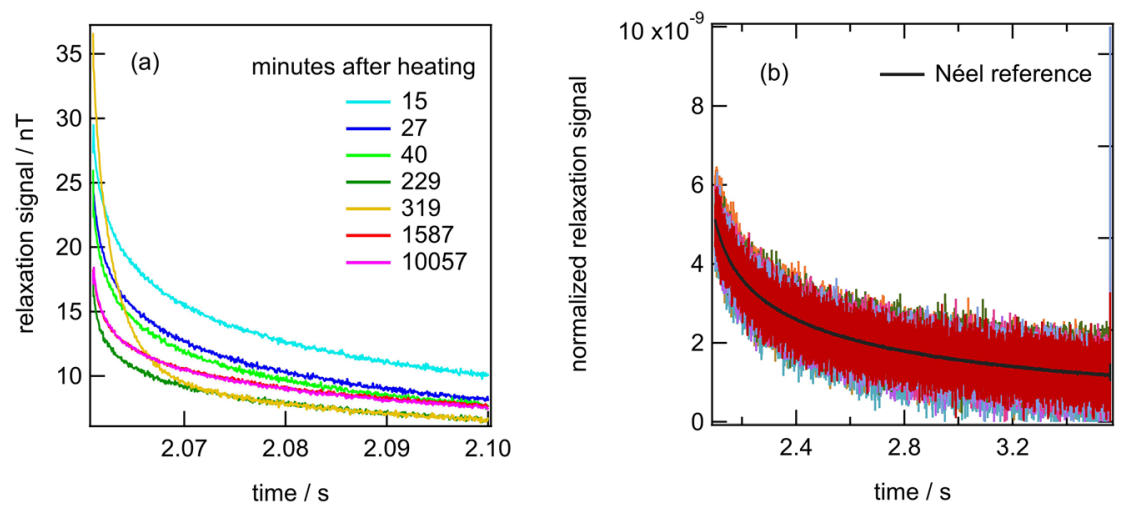

Figure 16: MRX curves measured on (a) 7.5 and (b) 10 wt $\%$ gelatin samples at different times of the gelation process.

measurements at higher ac field amplitudes or large-magnitude superimposed dc fields, the effect of magnetic field amplitude on the Brownian relaxation time has to be separated. The magnetic field-dependence of the Brownian relaxation time was investigated by measuring the ACS spectra with the setup originally developed for measuring the dynamics in a rotating magnetic field. Experimental details were briefly summarized in Section 2.

Experimental studies of the dependence of Brownian and Néel relaxation times of iron-oxide single-core MNPs (SHP-20 and SHP-25 from Ocean Nanotech) on ac field amplitude and on a superimposed dc magnetic field-either parallel or perpendicular to the ac probing field-were presented in the study by Dieckhoff et al. [40]. It was demonstrated that Equations (20) and (21) can well be applied. In order to extend the range of the Langevin parameters $\xi$, ACS measurements were also performed at $\mathrm{Ni}$ nanorods [52]. Combining their magnetic moment of the order of $10^{-17} \mathrm{Am}^{2}$ and the maximum field amplitude of $9 \mathrm{mT}$, which can be applied with the given setup, Langevin parameters up to 80 are covered. For comparison, the spherical $\mathrm{CoFe}_{2} \mathrm{O}_{4}$ nanoparticles used for the nanorheological studies have magnetic moments of the order of $7 \cdot 10^{-19} \mathrm{Am}^{2}$, providing a Langevin parameter at room temperature and $9 \mathrm{mT}$ of approximately 1.5 . Caused by shape anisotropy, these Ni nanorods with lengths of about $270 \mathrm{~nm}$ are strongly blocked so that dynamics are solely determined by the Brownian mechanism.

Figure 17(a) depicts the spectra of the imaginary part measured on an aqueous suspensions of Ni nanorods for different amplitudes of the ac magnetic field. To reduce dipolar interactions between nanorods, the nanorods volume fraction amounted to $5 \cdot 10^{-5}$. With increasing ac field amplitude, the position of the maximum shifts to higher frequencies and at the same its magnitude decreases. With the assumption that the maximum occurs at $\omega \tau_{B}=1$, Equation (20) can be applied to fit the dependence of the 

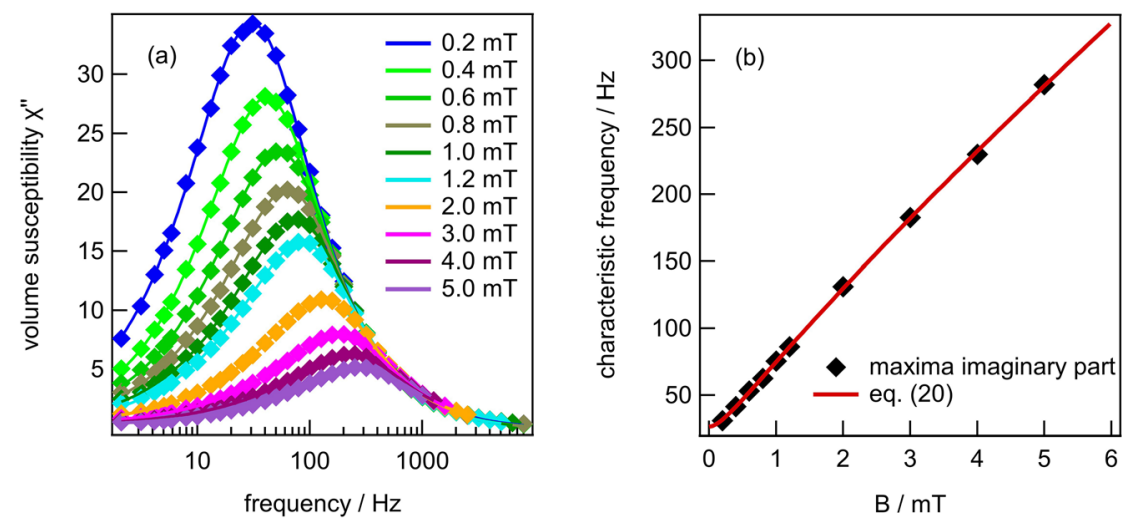

Figure 17: (a) Imaginary part versus frequency measured on Ni nanorod sample for different amplitudes of the ac magnetic field. (b) Extracted characteristic frequency versus field amplitude. Solid line shows fit with Equation (20) and assuming $\omega_{c h} \tau_{B}=1$.

characteristic frequency $f_{c h}$ on ac magnetic field amplitude. The best fit to the experimental data is shown in Figure 17(b). As can be seen, Equation (20) can be applied for the ac magnetic field amplitude dependence of the Brownian relaxation time over a large range of Langevin parameters.

In order to determine the effect of an oscillating magnetic field-as applied by Tschöpe et al. [22, 39] for their nanorheological studies using magneto-optical detection-on the Brownian relaxation time, we also performed ACS measurements in a small ac magnetic field and a perpendicular static magnetic field with magnitudes up to $9 \mathrm{mT}$ on the aqueous Ni nanorod suspension. The characteristic frequency versus static magnetic field magnitude is shown in Figure 18. Note that fitting the characteristic frequency (or Brownian relaxation time) with Equation (21) with the two parameters $\xi$ and $t_{\mathrm{B}}$ is only unique at low $\xi$ values. Therefore, the dashed line in Figure 18 was calculated with the parameters from the fit of the data in Figure 17(b). The good agreement between experimental results and model indicates that Equation (21) can be applied to determine the effect of a perpendicularly applied static magnetic field on the Brownian relaxation time. This is important for the determination of rheological parameters from ACS measurements since they are included only in the zero-field Brownian relaxation time $t_{\mathrm{B}}$.

\section{Discussion}

The use of thermally blocked MNPs as local probes for nanorheological investigations is straight forward for viscous media. The Brownian relaxation time $\tau_{\mathrm{B}}$, which is proportional to the dynamic viscosity, can be determined by any dynamic magnetic 


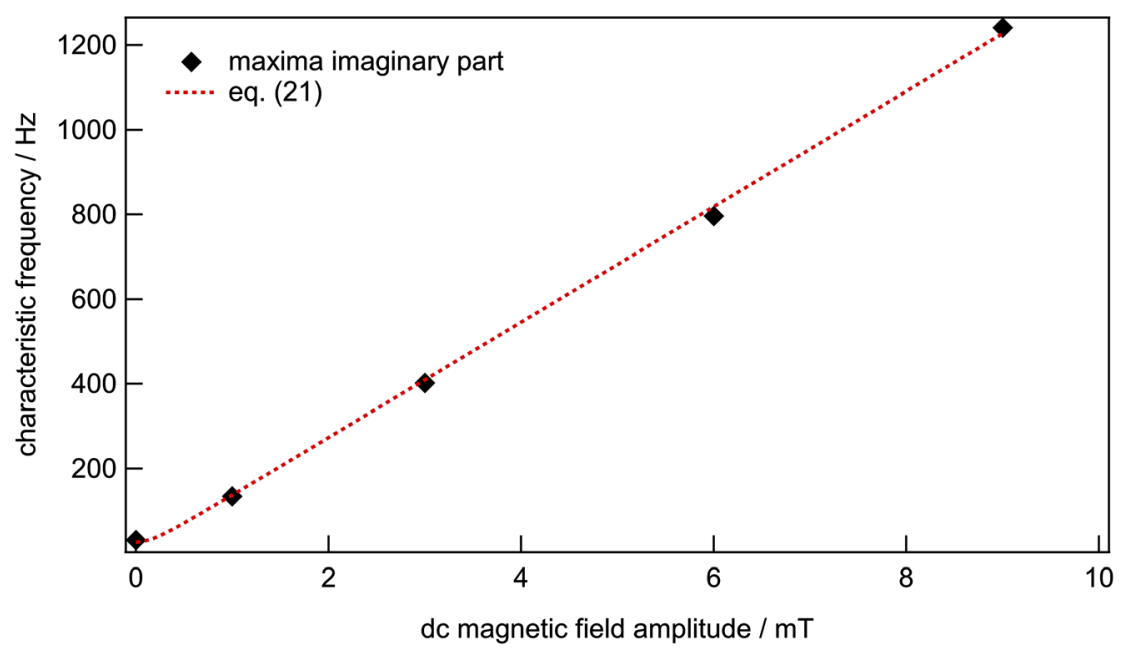

Figure 18: Dependence of the characteristic frequency on the magnitude of the perpendicular static magnetic field magnitude. Dashed line shows dependence calculated with Equation (21) using the parameters for $\xi$ and $\tau_{B}$ from Figure 17(b).

measurement method. Here we focused on measurements of the complex (ac) susceptibility and the magnetorelaxation signal. The advantage of ACS is that a rather large range of relaxation times can be covered, while MRX measurements are generally restricted to relaxation times above a few $100 \mu$ s.

The situation is more complex for viscoelastic media. As we have demonstrated for the cases of a Maxwell and a Voigt-Kelvin model as the two limiting cases, viscosity and shear modulus have a very different effect on the ac susceptibility spectrum. A qualitative analysis of the ACS spectrum already gives some indication on the dominating viscoelastic model. The ACS spectrum of blocked MNPs in any viscoelastic matrix can basically be described by Equation (12). In contrast to numerical models for a Maxwell [42] or Voigt-Kelvin system [41], the computational effort is much smaller. The analysis procedure is straight forward for systems without parameter distributions: Using measured values of real and imaginary parts, the complex shear modulus or complex viscosity can be calculated as a function of frequency. If distributions (e.g., of the hydrodynamic size) have to be considered, the equation system is still solvable, proposed that the distribution-as generally determined from a fit of the ACS spectrum measured on a sample with MNPs in a Newtonian fluid with known viscosity-does not change. For the temporal evolution of the gelation process, as studied in this contribution, this is certainly a coarse assumption.

Comparing ACS and MRX for nanorheological studies applying MNPs as local probes, ACS has the advantage that there are theoretical models for Maxwell, VoigtKelvin and Jeffrey systems [41-43] describing the complex susceptibility spectrum. 
As we have described in Section 3.2, the Debye model can easily be modified for nonNewtonian media by inserting a complex viscosity/shear modulus. In contrast, for the analysis of MRX data, there are currently only expressions for the effective relaxation time, which depend on both viscosity and shear modulus, i.e., their independent determination from a single MRX curve is not possible. For comparison, in an ACS measurement, real and imaginary parts of the complex susceptibility as a function of frequency can be used for the independent extraction of rheological parameters.

In conventional rheology, the relationship between stresses and strains is measured in order to determine the complex shear modulus. As a result, frequencydependent values for storage $G$ ' and loss modulus $G$ ' are obtained. In Section 4.2, we have demonstrated (similar results were obtained by Tschöpe et al. [22]) that experimental spectra could only be fitted by implementing a distribution of (local) dynamic viscosity. The main difference between conventional rheology and nanorheology is that MNPs are used in the latter one as probes for the local (nano)environment. Ignoring interactions between MNPs, the measured ACS spectrum is the superposition of the contributions from the individual nanoparticles inside the sample. Since-in a complex matrix as an aqueous gelatin solution-each MNP senses its own local environment, measured spectra cannot be described by a single value of complex viscosity (or shear modulus). Injecting MNPs as local probes into a matrix, the MNPs' response crucially depends on the way they are embedded in the matrix and on their size compared to the characteristic length scales of the matrix material [24, 53]. For example, if the nanoprobes are larger than the mesh size of a gel network, their response is expected to reflect the macrorheological properties of the matrix [22]. If they are "swimming" inside a mesh, they will sense the viscosity of the liquid. Thus, dynamic magnetic measurement techniques, such as ACS, provide more information than just the rheological parameters of the matrix on the nanoscale. Also, their dynamics strongly depends on whether they are functionalized so that they can be directly linked to the surrounding matrix $[54,55]$.

While magnetoviscous effects-caused by dipolar interactions and thus structure formation-were observed in conventional rheology [28], we did not observe any indications on magnetoviscous effects in dynamic magnetic measurements. Importantly, one should keep in mind that the magnetoviscous effect as theoretically proposed by Shliomis [27] does not occur in a dynamic magnetic measurement with stationary sample and that the Brownian relaxation time itself depends on magnetic field strength. For all samples investigated so far, the change of the ac susceptibility spectrum with magnetic field amplitude could well be described by the fielddependent Brownian relaxation time. Performing ac susceptibility measurements on samples with significantly higher MNP concentrations-as, e.g., done by Fischer et al. [56], the additional problem arises that dipolar interactions directly causes a shift of the ACS spectrum toward lower frequency $[57,58]$, thus pretending a magnetoviscous effect. 


\section{Conclusions}

Dynamic magnetic measurement techniques such as ACS and MRX in combination with thermally blocked MNPs are a powerful tool to study the interaction between MNPs and the surrounding matrix. Applying appropriate models, such measurements can be used to determine rheological parameters on the nanoscale. However, one should keep in mind that these parameters, extracted from the MNP dynamics, may not be the same as the ones obtained from conventional rheology. But since MNPs sense their local environment on the nanoscale, dynamic magnetic measurements provide important information on the MNPs' embedding in and interaction with the matrix.

This contribution focused on ACS and MRX measurements. However, similarly, the dynamic magnetic response of MNPs can also be studied in oscillating-as performed by Tschöpe et al. [22, 39]-or rotating magnetic fields.

The methodology described in this contribution can be applied to different matrix systems, as, e.g., cells in biomedicine, other gel-like materials such as xanthan [23] and liquid crystals.

Acknowledgment: Financial support by the Deutsche Forschungsgemeinschaft, DFG Priority Program 1681 (LU800/4-1, LU800/4-2, LU800/4-3) is gratefully acknowledged. Author contribution: All the authors have accepted responsibility for the entire content of this submitted manuscript and approved submission.

Research funding: None declared.

Conflict of interest statement: The authors declare no conflicts of interest regarding this article.

\section{References}

1. Brown WF Jr. Thermal fluctuations of a single-domain particle. Phys Rev 1963;130:1677-86.

2. Coffey WT, Cregg PJ, Kalmykov YP. On the theory of Debye and Néel relaxation of single domain ferromagnetic particles. Adv Chem Phys 1993;83:263-464.

3. Néel L. Some theoretical aspects of rock-magnetism. Adv Phys 1955;4:191-243.

4. Raikher YL, Shliomis MI. The effective field method in the orientational kinetics of magnetic fluids and liquid crystals. Adv Chem Phys 1994;87:595-751. 1994.

5. Fannin PC, Charles SW. On the calculation of the Néel relaxation time in uniaxial single-domain ferromagnetic particles. J Phys D Appl Phys 1994;27:185-8.

6. Eberbeck D, Wiekhorst F, Steinhoff U, Trahms L. Aggregation behaviour of magnetic nanoparticle suspensions investigated by magnetorelaxometry. J Phys Condens Matter 2006;8:S2829-46.

7. Ludwig F, Heim E, Schilling M. Characterization of magnetic nanoparticles by analyzing the magnetization and relaxation dynamics using fluxgate magnetometers. J Appl Phys 2007;101: 113909.

8. Martsenyuk MA, Raikher YL, Shliomis MI. On the kinetics of suspensions of ferromagnetic particles. Sov Phys JETP 1974;38:413-6. 
9. Fannin PC, Scaife BKF, Charles SW. The measurement of the frequency dependent susceptibility of magnetic colloids. J Magn Magn Mater 1988;72:95-108.

10. Ahrentorp F, Astalan AP, Jonasson C, Blomgren J, Qi B, Thompson Mefford O, et al. Sensitive high frequency ac susceptometers in magnetic nanoparticle applications. AIP Conf Proc 2010;1311: 213-23.

11. Ludwig F, Eberbeck D, Löwa N, Steinhoff U, Wawrzik T, Schilling M, et al. Characterization of magnetic nanoparticle systems with respect to their magnetic particle imaging performance. Biomed Eng 2013;58:535-45.

12. Eberbeck D, Wiekhorst F, Steinhoff U, Trahms L. Quantification of biomolecule agglutination by magnetorelaxometry. Appl Phys Lett 2009;95:213701.

13. Heim EFL, Schilling M. Binding assays with streptavidin-functionalized superparamagnetic nanoparticles and biotinylated analytes using fluxgate magnetorelaxometry. J Magn Magn Mater 2009;321:1628-31.

14. Dieckhoff JMS, Ludwig F. Protein detection with magnetic nanoparticles in a rotating magnetic field. J Appl Phys 2014;115:024701.

15. Remmer H, Dieckhoff J, Schilling M, Ludwig F. Suitability of magnetic single- and multi-core nanoparticles to detect protein binding with dynamic magnetic measurement techniques. J Magn Magn Mater 2015;380:236-40.

16. Astalan AP, Ahrentorp F, Johansson C, Larsson K, Krozer A. Biomolecular reactions studied using changes in brownian rotation dynamics of magnetic particles. Biosens Bioelectron 2004;19: 945-51.

17. Chung SH, Hoffmann A, Bader SD, Liu C, Makowski L, Chen L. Biological sensors based on brownian relaxation of magnetic nanoparticles. J Appl Phys 2004;85:2971-3.

18. Schrittwieser S, Pelaz B, Parak WJ, Lentijo-Mozo S, Soulantica K, Dieckhoff J, et al. Homogeneous biosensing based on magnetic particle labels. Sensors 2016;16:828.

19. Enpuku K, Shibakura M, Arao Y, Mizoguchi T, Kandori A, Hara M, et al. Wash-free detection of C-reactive protein based on third-harmonic signal measurement of magnetic markers. Jpn J Appl Phys 2018;57:090309.

20. Bacri J-C, Dumas J, Gorse D, Perzynski R, Salin D. Ferrofluid viscometer. J Phys Lattr 1985;46: L1199-1205. https://doi.org/10.1051/jphyslet:0198500460240119900.

21. Roeben E, Roeder L, Teusch S, Effertz M, Deiters UK, Schmidt AM. Magnetic particle nanorheology. Colloid Polym Sci 2014;292:2013-23.

22. Tschöpe A, Birster K, Trapp B, Bender P, Birringer R. Nanoscale rheometry of viscoelastic soft matter by oscillating field magneto-optical transmission using ferromagnetic nanorod colloidal probes. J Appl Phys 2014;116:184305.

23. Sriviriyakula T, Bogrena S, Schaller V, Jonasson C, Blomgren J, Ahrentorp F, et al. Nanorheological studies of xanthan/water solutions using magnetic nanoparticles. J Magn Magn Mater 2019;473: 268-71.

24. Hess M, Rochels P, Roeben E, Zylla M, Webers S, Wende H, et al. Size effects on rotational particle diffusion in complex fluids as probed by magnetic particle nanorheology. Phys Chem Chem Phys 2019;21:26525-39.

25. Remmer H, Dieckhoff J, Tschöpe A, Roeben E, Schmidt AM, Frank L. Dynamics of $\mathrm{CoFe}_{2} \mathrm{O}_{4}$ singlecore nanoparticles in viscoelastic media. Phys Procedia 2017;75:1150-7.

26. Odenbach S. Magnetoviscous effects in ferrofluids. Berlin: Springer; 2002.

27. Shliomis MI. Effective viscosity of magnetic suspensions. Sov Phys JETP 1977;34:1291-4.

28. Nowak J, Wolf D, Odenbach S. A rheological and microscopical characterization of biocompatible ferrofluids. J Magn Magn Mater 2014;354:98-104.

29. Kötitz R, Matz H, Trahms L, Koch H, Weitschies W, Rheinländer T, et al. SQUID based remanence measurements for immunoassays. IEEE Trans Supercon 1997;7:3678-81. 
30. Matz H, Drung D, Hartwig S, Groß H, Kötitz R, Müller W, et al. A SQUID measurement system for immunoassays. Appl Supercond 1998;6:577-83.

31. https://qd-europe.com/de/en/product/materials-science/magnetometers/ac-susceptometer.

32. https://qd-europe.com/de/en/product/materials-science/magnetometers/mpms3-squidmagnetometer.

33. Dieckhoff J, Schilling M, Ludwig F. Fluxgate based detection of magnetic nanoparticle dynamics in a rotating magnetic field. Appl Phys Lett 2011;99:112501.

34. Shliomis $M$, Stepanov V. Frequency dependence and long time relaxation of the susceptibility of the magnetic fluids. J Magn Magn Mater 1993;122:176-81.

35. Svedlindh P, Jonsson T, Garcia- Palacios JL. Intra-potential-well contribution to the AC susceptibility of a noninteracting nano-sized magnetic particle system. J Magn Magn Mater 1997; 169:323-34.

36. Ludwig F, Balceris C, Johansson C. The anisotropy of the AC susceptibility of immobilized magnetic nanoparticles-the influence of intra-potential well contribution on the AC susceptibility spectrum. IEEE Trans Magn 2017;53:6101004.

37. Ludwig F, Balceris C, Jonasson C, Johansson C. Analysis of AC susceptibility spectra for the characterization of magnetic nanoparticles. IEEE Trans Magn 2017;53:6100904.

38. DiMarzio EA, Bishop M. Connection between the macroscopic electric and mechanical susceptibilities. J Chem Phys 1974;60:3802-11.

39. Gratz M, Tschöpe A. Size effects in the oscillatory rotation dynamics of Ni nanorods in poly(ethylene oxide) solutions. Macromolecules 2019;52:6600-12.

40. Dieckhoff J, Eberbeck D, Schilling M, Ludwig F. Magnetic-field dependence of Brownian and Néel relaxation times. J Appl Phys 2016;119:043903.

41. Raikher YL, Rusakov VV, Coffey WT, Kalmikov YP. Dynamic susceptibilities of an assembly of dipolar particles in an elastic environment. Phys Rev E 2001;63:031402.

42. Raikher YL, Rusakov VV. Orientational kinetics of dipolar particles in a Maxwell fluid matrix: inertialess limit for the rotary microrheology. Phys Rev B 2005;72:061406.

43. Rusakov VV, Raikher YL. Magnetic response of a viscoelastic ferrodispersion: from a nearly Newtonian ferrofluid to a Jeffreys ferrogel. J Chem Phys 2017;147:124903.

44. Remmer H, Roeben E, Schmidt AM, Schilling M, Ludwig F. Dynamics of magnetic nanoparticles in viscoelastic media. J Magn Magn Mater 2017;427:331-5.

45. Rusakov VV, Raikher YL. Orientational brownian motion in a viscoelastic medium. Colloid J 2017; 79:264-9.

46. Yoshida T, Enpuku K. Simulation and quantitative clarification of AC susceptibility of magnetic fluid in nonlinear brownian relaxation region. Jpn J Appl Phys 2009;48:127002.

47. Gratz M, Tschöpe A. Optical transmission versus ac magnetization measurements for monitoring colloidal Ni nanorod rotational dynamics. J Phys D Appl Phys 2017;50:015001.

48. Fock J, Balceris C, Costo R, Zeng L, Ludwig F, Hansen MF. Field-dependent dynamic responses from dilute magnetic nanoparticle dispersions. Nanoscale 2018;10:2052-66.

49. Cheng N-S. Formula for the viscosity of a glycerol-water mixture. Ind Eng Chem Res 2008;47: 3285-8.

50. Ludwig F, Wawrzik T, Yoshida T, Gehrke N, Briel A, Eberbeck D, et al. Optimization of magnetic nanoparticles for magnetic particle imaging. IEEE Trans Magn 2012;48:3780-3.

51. Normand V, Muller S, Ravey J-C, Parker A. Gelation kinetics of gelatin: a master curve and network modeling. Macromolecules 2000;33:1063-71.

52. Remmer H, Gratz M, Tschöpe A, Ludwig F. Magnetic field dependence of Ni nanorod brownian relaxation. IEEE Trans Magn 2017;53:6101204.

53. Cai L-H, Panyukov S, Rubinstein M. Mobility of nonsticky nanoparticles in polymer liquids. Macromolecules 2011;44:7853-63. 
54. Roeder L, Bender P, Kundt M, Tschöpe A, Schmidt AM. Magnetic and geometric anisotropy in particle-crosslinked ferrohydrogels. Phys Chem Chem Phys 2015;17:1290-8.

55. Landers J, Roeder L, Salamon S, Schmidt AM, Wende H. Particle-matrix interaction in cross-linked PAAm-hydrogels analyzed by mössbauer spectroscopy. J Phys Chem C 2015;119:20642-8.

56. Fischer B, Wagner J, Schmitt M, Trieu V, Hempelmann R. Dependence of brownian relaxation on the volume fraction and an external magnetic field. Z Phys Chem 2006;220:69-77.

57. Ivanov AO, Zverev VS, Kantorovich SS. Revealing the signature of dipolar interactions in dynamic spectra of polydisperse magnetic nanoparticles. Soft Matter 2016;12:3507-13.

58. Ivanov AO, Camp PJ. Theory of the dynamic magnetic susceptibility of ferrofluids. Phys Rev E 2018; 98:050602(R). 